\title{
Retroperitoneal LESS Donor Nephrectomy
}

\author{
A. van der Merwe, A. Bachmann, C. F. Heyns
}

Department of Urology (AVM, CFH), University of Stellenbosch and Tygerberg Hospital, Cape Town, South Africa and Department of Urology (AB), University Hospital Basel, Basel, Switzerland

\begin{abstract}
Donor nephrectomy with laparo-endoscopic single site (LESS) surgery has been reported via the transperitoneal approach. We describe a novel technique of retroperitoneal donor nephrectomy using a single surgical incision in the groin, below the abdominal skin crease or "bikini line". The LESS groin incision offers superior cosmesis, while the retroperitoneal approach has distinct advantages, such as the ability to identify the renal vessels early. The new procedure has been performed in two obese patients (body mass index 32 and $33 \mathrm{~kg} / \mathrm{m}^{2}$, respectively). The operative times were 4 and 5 hours, warm ischemic times 135 and 315 seconds, blood loss 100 and $250 \mathrm{~mL}$, and hospitalization 3 and 2 days, respectively. Retroperitoneal LESS donor nephrectomy through a single, inconspicuous groin incision is feasible and safe. Further evaluation of the technique in a larger patient cohort is indicated.
\end{abstract}

Key words: laparoscopy; nephrectomy; transplant; LESS

Int Braz J Urol. 2010; 36: 602-8

\section{INTRODUCTION}

The first laparoscopic live donor nephrectomy was reported by Ratner et al. in 1995 and retroperitoneal endoscopic donor nephrectomy was described by Yang et al. in the same year $(1,2)$.

Laparo-endoscopic single site (LESS) donor nephrectomy is in its infancy and the optimal technique has yet to be established. The addition of needle ports or 3-5 $\mathrm{mm}$ ports (hybrid LESS) may make surgery easier, but may also defeat the purpose of improved cosmesis and less postoperative pain. Multichannel ports and novel bent instruments are available to overcome the lack of triangulation in single port surgery.

The complexity of the LESS approach should not be underestimated: Rajan and Turna reviewed LESS urological surgery worldwide and concluded "scissoring" may be problematic and the technique still needs to be fully evaluated with regards to benefits over standard laparoscopic approaches (3). The difficulty as well as feasibility of these procedures are underlined by Desai et al. reporting one hundred LESS cases where one patient died postoperatively as a consequence of LESS surgery (4).

In transumbilical LESS donor nephrectomy (reported initially as e-NOTES) the incision is extended up to $3 \mathrm{~cm}$ on either side of the umbilicus (5). This is done just prior to extraction of the kidney after the renal vessels have been transected. The cosmetic result of a scar around the umbilicus may not be acceptable to all donors, especially young females. Moreover, not using the full length of the final extraction incision during the procedure itself defies 
a basic principle of surgery. Using the full length of the incision during retroperitoneal LESS surgery may allow for normal triangulation, an easier operation and a shorter learning curve.

One of the main advantages of retroperitoneal renal surgery is the ease of dissecting the renal hilum, even in obese donors. Combining the retroperitoneal route with LESS surgery as performed transperitoneally may be a significant advance in the search for the perfect donor operation. The authors have combined experience of more than 250 cases of retroperitoneal donor nephrectomy and felt it safe to proceed to single site surgery for this operation. One should be careful that this technique is only to be used by experienced retroperitoneoscopic surgeons for donor operations. As the instruments for these cases were available to us for a limited period we performed the next two cases on our donor list. No other cases have been attempted and we are in the process to further fully evaluate the technique prospectively.

\section{SURGICAL TECHNIQUE}

For right-sided donor nephrectomy, the patient is positioned in the left lateral position over a fully flexed operating table. The right hip is in mild extension to allow access to the single groin port. The patient is secured with strapping to the table, to allow maneuverability of the table during surgery. Instruments for laparoscopic as well as open surgery are kept at hand in case emergency conversion to open surgery is required (Table-1).

An incision $6 \mathrm{~cm}$ long is made in the groin, below the "bikini line". The incision is deepened with open surgical technique (muscle splitting) to enter the retroperitoneal space. The psoas muscle is identified and the space posterior to the kidney is developed as described by Bachmann et al. (6). An inflation balloon (PDB 1000 Covidien, Mansfield, MA) is deployed behind the kidney and inflated with $800 \mathrm{~mL}$ air (Figure-1).

The Gelport ${ }^{\mathrm{TM}}$ (Applied Medical, Rancho Santa Margarita, CA) is placed in the incision and carbon dioxide pressure is established at $12 \mathrm{~mm} \mathrm{Hg}$ via the central $10 \mathrm{~mm}$ camera port (Figure-2). Surgical adhesive film is centrally placed over the Gelport ${ }^{\mathrm{TM}}$ to control gas leak. Two $5 \mathrm{~mm}$ ports are placed on either side of the lens on the edges of the Gelport ${ }^{\mathrm{TM}}$.

Gerota's fascia is opened close to the psoas muscle to expose the fatty tissue harboring the inferior vena cava and renal vessels. The vena cava is followed cranially to the renal vein. The renal artery pulsation is identified to the left of this in the fatty tissue and dissected free. A $5.5 \mathrm{~mm} 30^{\circ}$ lens is used for the hilar dissection, so clashing between instruments is reduced. A curved fenestrated grasper is inserted via the Gelport ${ }^{\mathrm{TM}}$ inferior rim and is used to grasp the edge of Gerota's fascia to keep fat from obscuring the view.

The hilar vessels are dissected free to allow enough space for a vascular stapler to pass without

Table 1 - Instruments used for retroperitoneal LESS donor nephrectomy.

\begin{tabular}{|c|c|}
\hline Instrument & Use \\
\hline Gelport $^{\mathrm{TM}}$ - & Placed in groin incision to allow triangulation \\
\hline Bariatric bipolar grasper (Karl Storz ${ }^{\mathrm{TM}}$ ) & Needed for dissection on cranial part of hilum \\
\hline Bariatric scissors (Karl Storz ${ }^{\mathrm{TM}}$ ) & As above and to mobilise the upper pole \\
\hline Curved LESS grasper (Karl Storz ${ }^{\mathrm{TM}}$ ) & Portless via Gelport ${ }^{\mathrm{TM}}$ to retract fat \\
\hline Lens $10 \mathrm{~mm} 30^{\circ}$ normal length (Karl Storz ${ }^{\mathrm{TM}}$ ) & Initial part of operation \\
\hline 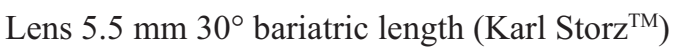 & Hilar dissection and vessel stapling \\
\hline 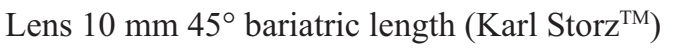 & Adrenal and upper pole dissection \\
\hline Large endo-bag (Covidien ${ }^{\mathrm{TM}}$ ) & To hold kidney up to expose pedicle for stapler \\
\hline TA 30 vascular stapler (Covidien ${ }^{\mathrm{TM}}$ ) & To occlude and cut renal artery and vein \\
\hline
\end{tabular}




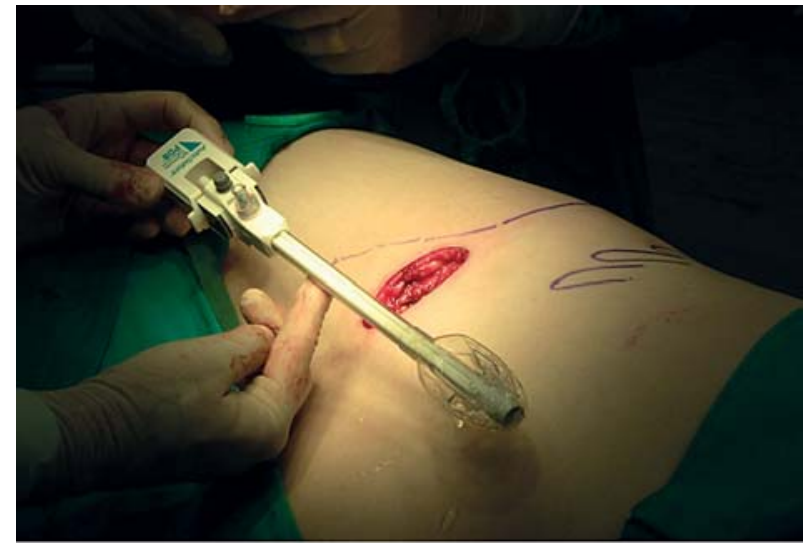

Figure 1 - An inflation balloon is inserted retroperitoneally via the $6 \mathrm{~cm}$ groin incision below the "bikini line".

catching tissue behind the vessels in the stapler jaws. The adrenal is dissected from the kidney using the 10 $\mathrm{mm} 45^{\circ}$ lens. The adrenal artery is clipped close to the renal artery to prevent it from hanging in the potential staple line. The ureteric dissection is completed, preserving maximal tissue around the ureter, which is clipped and cut distally.

The kidney is freed from the peri-renal fat using the $45^{\circ}$ lens for the upper pole and using the curved grasper to allow counter-traction on the perirenal fat. Once the kidney is completely free the renal vessels are inspected for safety prior to stapling. The $5.5 \mathrm{~mm} 30^{\circ}$ bariatric lens is used and rotated $180^{\circ}$ to

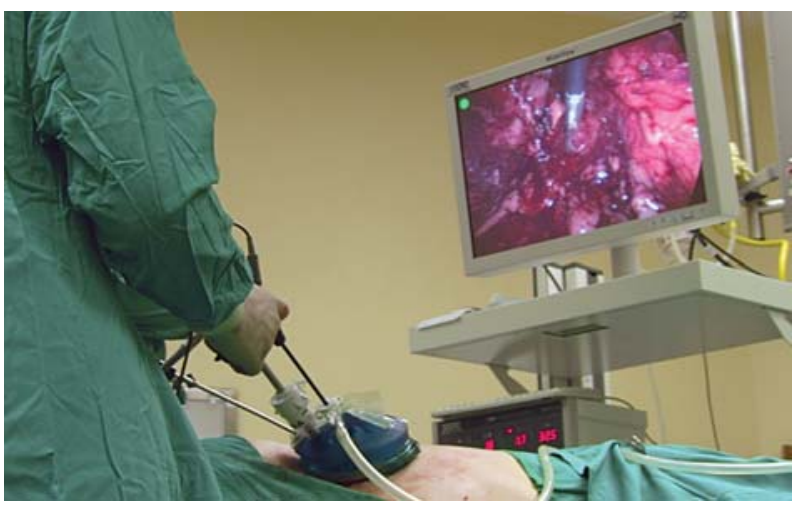

Figure 2 - The Gelport ${ }^{\mathrm{TM}}$ in use. Note that the surgeon stands on the abdominal side of the patient, which is unusual for retroperitoneal surgery. visualize the hilum maximally and reduce clashing due to the small diameter of the lens.

A $15 \mathrm{~mm}$ large laparoscopic retrieval bag (EndoCatch II, Covidien, Mansfield, MA) is inserted via the right side of the Gelport ${ }^{\mathrm{TM}}$ (without the use of an extra port), deployed over the kidney and closed partially to expose the hilum. Great care is taken to protect the vessels from the plastic covered metal rim of the bag as described elsewhere (7).

A 5-12 mm port (Applied Medical, Rancho Santa Margarita, California) is placed on the left side of the Gelport to allow a vascular stapler as well as the laparoscopic scissors easy and rapid exchange. The kidney is elevated with the retrieval bag (with the ureter visible and free) and the renal artery is stapled (TA 30 linear stapler - Covidien, Mansfield, MA) and cut with laparoscopic scissors. The renal vein is managed in similar fashion. The kidney is removed together with the outer covering of the Gelport $^{\mathrm{TM}}$ and processed immediately in slushed ice and Euro-Collins solution. Furosemide and mannitol are not routinely administered intravenously prior to harvesting.

The procedure has been performed on two obese male donors (BMI $32 \mathrm{~kg} / \mathrm{m}^{2}$ and $33 \mathrm{~kg} / \mathrm{m}^{2}$, respectively). Routine extensive workup was done and informed consent was obtained. In both cases the right kidney was harvested, as it appeared more suitable on preoperative CT angiography. The patients were placed under general anesthesia with mechanical ventilation and full muscle relaxation to enlarge the retroperitoneal working space maximally.

Surgical time was 4 hours and 5 hours, respectively. Warm ischemic times (measured from the time of renal artery occlusion until fluid is seen exiting from the renal vein during perfusion with cold Euro-Collins solution) were 135 seconds and 315 seconds, respectively (the latter time was prolonged due to dislodgement of the camera port from the Gelport $\left.{ }^{\mathrm{TM}}\right)$. Blood loss was $100 \mathrm{~mL}$ and $250 \mathrm{~mL}$ (calculated and recorded by the attending anesthetist). Hospital stay was 3 and 2 days, respectively. Both patients were pain-free on discharge. The postoperative result after removal of the wound drain is shown in Figure-3. Both kidneys functioned well postoperatively. 


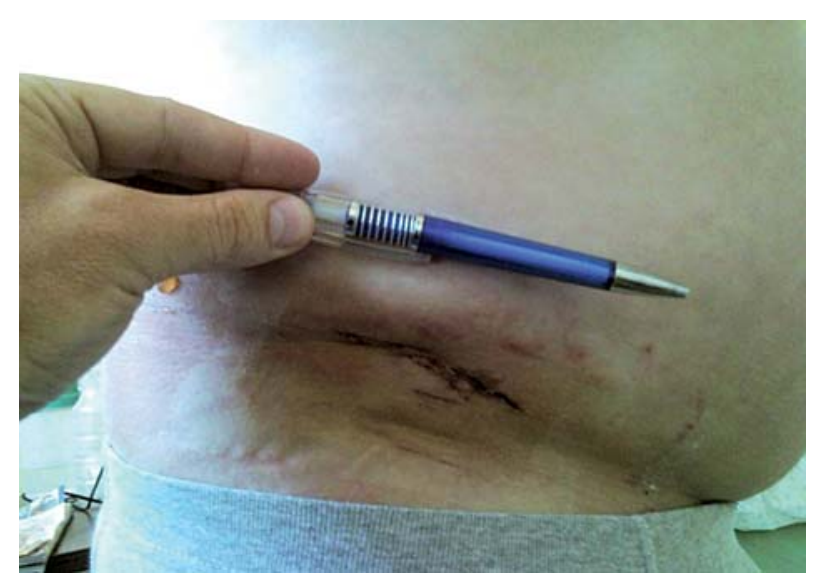

Figure 3 - Groin incision at discharge of patient on postoperative day 3 .

\section{COMMENTS}

A recent report described live donor nephrectomy using pure LESS surgery via a high Pfannenstiel incision and transperitoneal route (8). The authors elevated a flap of skin to allow triangulation and exchanged ports for dissection and stapling. While this is an ingenious novel approach, the angle from the midline lower abdominal incision to the upper pole and hilum of the kidney may be difficult, especially in more obese patients. The same team reported a matched comparison (retrospectively) between standard laparoscopy and their Pfannenstiel LESS approach. No difference in postoperative pain, hospital stay or analgesic requirements were found between the two groups. This suggested cosmesis may be the only difference (9). However, we believe the elevation of flaps (in contrast to our retroperitoneal LESS approach) may contribute to increased pain postoperatively.

The Gelport ${ }^{\mathrm{TM}}$ used in the new technique described above allows for multiple atraumatic port exchanges and reduces warm ischemic time, as the opening in the abdominal wall is created at the start of surgery. It utilizes the full length of the incision during surgery and can be seen as an advance, compared to the use of a multi-port device with lack of triangulation, and extension of the incision at the time of graft extraction as described by Gill et al. (5).
A novel approach quite similar to ours were used by Rye et al. where they used the base of the Gelport $^{\mathrm{TM}}$, the Alexis ${ }^{\mathrm{TM}}$ ring, to affix a surgical glove and use the fingers as port sites in 14 successful urological procedures (10).

Fatal complications related to living donor nephrectomy are associated with both locking- and nonlocking surgical clips (11). The potential safety of LESS is probably comparable to open surgery and ordinary laparoscopic surgery, and is primarily related to the technique used for occlusion of the renal vessels.

In the technique described by Gill et al. (5) the donor kidney is pulled up against the abdominal wall while the skin incision is enlarged to allow removal of the kidney. The pressure on the kidney and the prolonged warm ischemic time may be detrimental to renal function. The Gelport ${ }^{\mathrm{TM}}$ LESS technique described above has the advantage that no further incisions are made after the initial dissection.

Canes et al. performed a matched-pair comparison of LESS versus standard laparoscopic left donor nephrectomy and found significantly longer warm ischemic times in the LESS group (12). This is probably related to additional time required for extension of the incision after the renal vessels have been severed and the donor kidney is being extracted.

The superior cosmetic result achieved by retroperitoneal LESS surgery may contribute significantly to the recruitment of more kidney donors. The in-hospital pain scores are similar after LESS compared with laparoscopic live donor nephrectomy, but when out-of-hospital pain scores are reviewed, LESS donors report significantly less pain and quicker return to $100 \%$ function (12).

\section{CONFLICT OF INTEREST}

Karl Storz supplied the instruments and Surgical Innovations SA the Gelports ${ }^{\mathrm{TM}}$ and ports.

\section{REFERENCES}

1. Ratner LE, Ciseck LJ, Moore RG, Cigarroa FG, Kaufman HS, Kavoussi LR: Laparoscopic live donor nephrectomy. Transplantation. 1995; 60: 1047-9. 
2. Yang SC, Park DS, Lee DH, Lee JM, Park K: Retroperitoneal endoscopic live donor nephrectomy: report of 3 cases. J Urol. 1995; 153: 1884-6.

3. Rajan P, Turna B: New trends in minimally invasive urological surgery. Int Braz J Urol. 2009; 35: 51420.

4. Desai MM, Berger AK, Brandina R, Aron M, Irwin BH, Canes D, et al.: Laparoendoscopic single-site surgery: initial hundred patients. Urology. 2009; 74: 805-12.

5. Gill IS, Canes D, Aron M, Haber GP, Goldfarb DA, Flechner S, et al.: Single port transumbilical (ENOTES) donor nephrectomy. J Urol. 2008; 180: 63741; discussion 641.

6. Bachmann A, Dickenmann M, Gürke L, Giannini O, Langer I, Gasser TC, et al.: Retroperitoneoscopic living donor nephrectomy: a retrospective comparison to the open approach. Transplantation. 2004; 78: 16871.

7. Bollens R, Mikhaski D, Espinoza BP, Rosenblatt A, Hoang AD, Abramowicz D, et al.: Laparoscopic live donor right nephrectomy: a new technique to maximize the length of the renal vein using a modified Endo GIA stapler. Eur Urol. 2007; 51: 1326-31.

\section{Correspondence address:}

Dr. André van der Merwe

Department of Urology, PO Box 19063

University of Stellenbosch \& Tygerberg Hospital

Tygerberg 7505, Cape Town, South Africa

Fax: + 2721 933-8010

E-mail: arvdm@sun.ac.za
8. Andonian S, Herati AS, Atalla MA, Rais-Bahrami S, Richstone L, Kavoussi LR: Laparoendoscopic singlesite pfannenstiel donor nephrectomy. Urology. 2010; 75: 9-12.

9. Andonian S, Rais-Bahrami S, Atalla MA, Herati AS, Richstone L, Kavoussi LR: Laparoendoscopic singlesite pfannenstiel versus standard laparoscopic donor nephrectomy. J Endourol. 2010; 24: 429-32.

10. Ryu DS, Park WJ, Oh TH: Retroperitoneal laparoendoscopic single-site surgery in urology: initial experience. J Endourol. 2009; 23: 1857-62.

11. Friedman AL, Peters TG, Jones KW, Boulware LE, Ratner LE: Fatal and nonfatal hemorrhagic complications of living kidney donation. Ann Surg. 2006; 243: 126-30.

12. Canes D, Berger A, Aron M, Brandina R, Goldfarb DA, Shoskes D, et al.: Laparo-endoscopic single site (LESS) versus standard laparoscopic left donor nephrectomy: matched-pair comparison. Eur Urol. 2010; 57: 95-101. 


\section{EDITORIAL COMMENT}

Since its original description in 1991, urologic laparoscopy has shown exponential growth (1). The laparoscopic technique has been associated with reduced postoperative pain, improved cosmesis, decreased hospital stay, and improved convalescence while maintaining equivalent outcomes to open surgery. As innovations have been incorporated, a natural progression has led to the evaluation of the number of ports required to safely perform laparoscopic procedures. This interest has intensified with the realization that a decrease in port size and number of ports could decrease operative morbidity leading to reduced narcotic requirements, bleeding risk, shorter hospital stay, a faster return to work, and improved cosmetic outcome (2).

While making the transition from conventional laparoscopy to Laparoendoscopic single-site (LESS) procedures, many technical challenges have been encountered (3). Inherent to the use of a common abdominal entry point is instrument crowding. This crowding leads to a loss of triangulation and tissue manipulation, mainstays in conventional laparoscopy, thus significantly increasing the technical difficulty of LESS procedures. Similarly, crowding leads to internal and external clashing of instruments and handles which is often noted as the most frustrating aspect of LESS.

The authors used the GelPort ${ }^{\mathrm{TM}}$ as the access platform, which can help to provide adequate spacing, triangulation and flexibility of port placement for LESS procedures. Another advantage is that the opening in the abdominal wall created at the start of surgery for device placement is also used for graft extraction.

The authors should be congratulated for their elegant work using alternative ways for donor nephrectomies. Andonian et al. (4) had already compared LESS Pfannenstiel donor nephrectomy with a contemporary series of standard laparoscopic donor nephrectomies, with no significant difference in terms of operative time, warm ischemia time (WIT), estimated blood loss, median length of stay and narcotic requirements. The Retroperitoneal LESS technique described by the authors has the advantages of retroperitoneal approach and better aesthetic appearance.
In our initial experience with LESS we performed an extraperitoneal radical nephroureterectomy through a $5 \mathrm{~cm}$ Gibson incision with Gelport and conventional instruments. Working space was obtained with open medial mobilization of the colon and the kidney before the location of Alexis' retractor. The surgeon's view in this procedure is inferior and distal to the renal pedicle. Pelvic ureter was dissected by the incision to improve the localization of anatomical landmarks after installation of pneumoretroperitoneum. Renal vessels could be controlled en bloc with an endoscopic stapler. Superior pole dissection was facilitated by distal traction of ureter. The kidney was removed through the incision and the bladder cuff was completed utilizing open technique and the same incision.

In donor nephrectomy, further caution must observed concerning WIT. In this present paper a higher operative and WIT compared to standard laparoscopy and LESS Pfannenstiel donor nephrectomy were reported. This can be adverse for the graft and for the donor. Although improved cosmesis is important, all efforts should be made to keep surgery as safe as possible either for donor or for recipient.

In our opinion, this novel technique should only be attempted by experienced laparoscopic surgeons who are comfortable in performing standard retroperitoneal laparoscopic donor nephrectomies.

LESS certainly represents the next step forward in the arena of minimally invasive surgery. However, for patients who undergo donor nephrectomy larger prospective randomized trials are needed to compare the postoperative pain levels, WIT, blood loss, operative time and graft function before it gains widespread acceptance.

\section{REFERENCES}

1. Clayman RV, Kavoussi LR, Soper NJ, Dierks SM, Merety KS, Darcy MD, Long SR, Roemer FD, Pingleton ED, Thomson PG: Laparoscopic nephrectomy. N Engl J Med. 1991; 324: 1370-1.

2. Raman JD, Bagrodia A, Cadeddu JA: Single-incision, umbilical laparoscopic versus conventional laparoscopic nephrectomy: a comparison of perioperative 
outcomes and short-term measures of convalescence. Eur Urol. 2009; 55: 1198-204.

3. Canes D, Desai MM, Aron M, Haber GP, Goel RK, Stein RJ, Kaouk JH, Gill IS: Transumbilical single-port surgery: evolution and current status. Eur Urol. 2008; 54: 1020-9.
4. Andonian S, Rais-Bahrami S, Atalla MA, Herati AS, Richstone L, Kavoussi LR: Laparoendoscopic singlesite Pfannenstiel versus standard laparoscopic donor nephrectomy. J Endourol. 2010; 24: 429-32.

\section{Dr. Walter Fernandes Correa \& Dr. Marcos Tobias-Machado Department of Urology $A B C$ Medical School and Brazilian Institute of Cancer Control São Paulo, SP, Brazil E-mail: tobias-machado@uol.com.br}

\section{EDITORIAL COMMENT}

In this manuscript, the authors propose a novel LESS approach using a surgical incision in the groin and report their experience with 2 obese patients. The authors are to be congratulated for exploring alternative ways for donor nephrectomies. Our group has recently described an alternative surgical technique that relies on the classic laparoscopic approach, supported by insertion of the surgeon's hand during kidney recovery using a lateral paramedian incision (1). We prefer a hand-assisted technique, because this approach is particularly useful when an incision is necessary to remove an intact surgical specimen in a short time after vessel division.

The LESS technique combines the advantages of retroperitoneal approach with the improved cosmesis and decreased discomfort. This experience represents a further effort to show that single port surgery is feasible and reproducible technique even in particular procedure such as living donor nephrectomy. Other Authors using NOTES technique are developing an incision-free procedure with graft delivery through a transvaginal incision in female donors with excellent aesthetic results (2).

However, the authors should proceed with caution when describing novel technique for renal recovery. While they have a wide experience with retroperitoneal donor nephrectomies, it should be emphasized that the approach described has to be attempted only by experienced laparoscopic surgeons who are comfortable in standard retroperitoneoscopic nephrectomies.
The rush for the development of new procedures carries the pitfall to publish papers with a low number of cases that may not represent the surgical reality. Moreover, taking in the account the difficulty and the risks of the procedure, the greater question that remains to be answered concerns the proper place of the LESS and NOTES approaches in urologic surgery. It is clear we can do this but far less clear that we should (3).

\section{REFERENCES}

1. Selvaggi FP, Ditonno P, Lucarelli G: Transperitoneal deviceless hand-assisted laparoscopic living donor nephrectomy: an alternative technique for kidney recovery. J Endourol. 2010. [Epub ahead of print]

2. Alcaraz A, Musquera M, Peri L, Transvaginal NotesAssisted laparoscopic living donor nephrectomy. Am J Tansplant.2010; 10 (suppl. 4); A219.

3. Strup SE. Editorial Comment on: Laparoendoscopic single-site Pfannenstiel donor nephrectomy. J Urol. 2010; 183: 1871; discussion 1872.

Dr. Pasquale Ditonno \& Dr. Giuseppe Lucarelli Department of Emergency and Organ Transplantation Urology Andrology and Kidney Transplantation Unit University of Bari Bari, Italy

E-mail:ditonno@urologia.uniba.it 